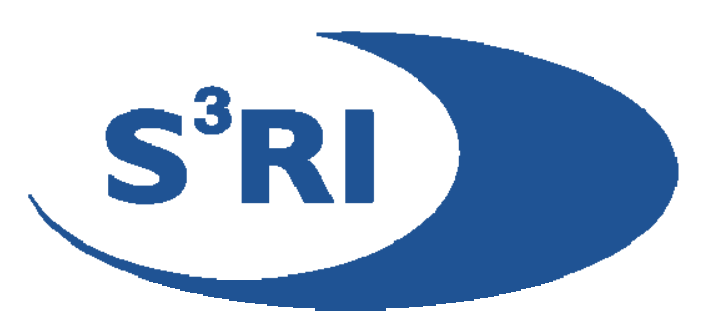

\title{
GEOGRAPHICALLY INTELLIGENT DISCLOSURE CONTROL FOR FLEXIBLE AGGREGATION OF CENSUS DATA
}

\section{Caroline Young, David Martin, Chris Skinner}

\begin{abstract}
This paper describes a geographically intelligent approach to disclosure control for protecting flexibly aggregated census data. Increased analytical power has stimulated user demand for more detailed information for smaller geographical areas and customized boundaries. Consequently it is vital that improved methods of statistical disclosure control are developed to protect against the increased disclosure risk. Traditionally methods of statistical disclosure control have been aspatial in nature. Here we present a geographically intelligent approach that takes into account the spatial distribution of risk. We describe empirical work illustrating how the flexibility of this new method, called local density swapping, is an improved alternative to random record swapping in terms of risk-utility.
\end{abstract}

\section{Southampton Statistical Sciences Research Institute Methodology Working Paper M07/12}




\title{
Geographically intelligent disclosure control for flexible aggregation of census data
}

\author{
CAROLINE YOUNG, DAVID MARTIN, CHRIS SKINNER
}

\begin{abstract}
This paper describes a geographically intelligent approach to disclosure control for protecting flexibly aggregated census data. Increased analytical power has stimulated user demand for more detailed information for smaller geographical areas and customized boundaries. Consequently it is vital that improved methods of statistical disclosure control are developed to protect against the increased disclosure risk. Traditionally methods of statistical disclosure control have been aspatial in nature. Here we present a geographically intelligent approach that takes into account the spatial distribution of risk. We describe empirical work illustrating how the flexibility of this new method, called local density swapping, is an improved alternative to random record swapping in terms of risk-utility.
\end{abstract}

Keywords: privacy, census data, spatial analysis, small area geography, quality issues

This is a preprint of an article submitted for consideration in the International Journal of Geographical Information Science ( [copyright Taylor and Francis]. International Journal of Geographical Information Science is available online at: http://www.informaworld.com/ 


\title{
GEOGRAPHICALLY INTELLIGENT DISCLOSURE CONTROL FOR FLEXIBLE AGGREGATION OF CENSUS DATA
}

\author{
CAROLINE YOUNG \\ (corresponding author: cjy@ soton.ac.uk)
}

School of Social Sciences, University of Southampton, Highfield, Southampton, SO17 1BJ

DAVID MARTIN

School of Geography, University of Southampton, Highfield, Southampton, SO17 1BJ

\section{CHRIS SKINNER}

School of Social Sciences, University of Southampton, Highfield, Southampton, SO17 1BJ 
International Journal of Geographical Information Science Submission

\section{Introduction}

Small area population data from censuses provide an important base layer in many GIS applications. Indeed, census geography played a key role in early GIS data structure development (Peucker and Chrisman, 1975). The ability to produce such detailed data is due to censuses' unique combination of detailed information about individuals and households with coverage of an entire population. However, achieving a complete or near complete response rate also makes the data highly susceptible to disclosure. Disclosure occurs when an individual can be identified in the data, leading to potentially sensitive information being revealed (Lambert, 1993). Protecting the confidentiality of census data by application of statistical disclosure control (SDC) methods is an integral part of the census process allowing use of protected data by researchers and policy makers across all sectors. SDC methods either restrict or modify the detail released (Willenborg and de Waal, 2001). Internationally, government statistical organizations undertake population data collection under various legislative frameworks (Holt 2003) which generally embody strict confidentiality requirements. The importance of the issue is magnified by reliance of official statistics on public trust in the safeguards employed. Historically, disclosure control could be handled by checking outputs manually before publication. However, in the past two decades, increases in computing power have stimulated increased demand on the part of census users, who are able to employ complex analytical techniques and process larger amounts of data. The growth of digital geographical information allows for the possibility of automatically generating census geographies as required (Openshaw and Rao 1995, Martin 2000). In fact, the provision of only one set of geographical units is no longer 
sufficient to meet users' demands. Many researchers require data which does not fit into neat aggregations of published zones. In the UK, for example, changes in health service organization creates demand for census data which cannot be matched by reaggregation of standard outputs. Such demand pressures have led to discussion of the development of flexible tabulation systems in, for example, the UK, Australia and the US (Rhind et al. 1991, Zayatz 2003, Duke-Williams and Rees, 1997). Any such system would allow users to create their own customised tables from unpublished individual records. In the absence of such systems, there is ongoing pressure for the production of outputs for multiple small area geography systems.

The disclosure risk facing statistical organizations is two-fold: first, the risk from outputs for any small population and, second, the additional risk from publishing multiple overlapping aggregations. There is particular demand for tables of counts at neighbourhood level but these are potentially risky since it may be possible to recognise data relating to particular individuals, especially in the light of local knowledge. Identification of individuals may lead to potentially sensitive information being revealed. The difficulty of multiple outputs is that published tables, although independently safe, may be compared with one another in order to reveal new information. This is particularly a problem when data are published for multiple geographical boundaries, described by DukeWilliams and Rees (1998) as geographical differencing. The response of the UK statistical organizations has been to publish counts only for hierarchical aggregations of the smallest output areas.

A closely related disclosure issue, arising from the availability of geographically referenced data, is termed geoprivacy and concerns the location of sensitive data at the 
International Journal of Geographical Information Science Submission

disaggregate level. While not usually considered in the context of aggregate census data, there is a close link to the central focus of this paper. Leitner and Curtis (2006) draw a distinction between statistical (attribute) and spatial (locational) confidentiality. Statistical confidentiality is associated with individual information, in GIS terms the equivalent of aspatial attributes, while spatial or locational confidentiality is concerned with the placement of individual-level statistical information on a map. To date, relatively little has been written about methods to protect the point mapping of individual information although this is of increasing concern to the GIS community. Geoprivacy is especially sensitive in studies of health and crime data. For example, law-enforcement agencies throughout the US provide crime maps (Leitner and Curtis, 2006), while point maps are often published representing cases of cancer or infectious diseases (for example, Zimmerman and Pavlik 2006, Armstrong et al. 1999). Leitner and Curtis (2006) note that an individual's residential location can be easily displayed, potentially leading to identification of the individual and disclosure of confidential information as inverse address matching technology can be used to reveal the street address and residents at a point location (Zimmerman and Pavlik, 2006).

The conventional approach to preserving spatial confidentiality in these data has been to adopt the same methodology as for census data, that is to aggregate records across populations large enough to ensure prevention of disclosure (Armstrong et al. 1999). However, aggregation damages the data, making research into causation with associated factors very difficult (Leitner and Curtis, 2006). Armstrong et al. (1999) introduced the term geographical masking (geomasking) for the modification of geographical coordinates to protect confidentiality. Methods include affine transformation and random perturbation. 
International Journal of Geographical Information Science Submission

Affine transformations relocate each point by change of scale, rotation, flipping or some concatenation of these masks. Random perturbation or jittering involves adding noise to original locations. According to Armstrong et al. (1999), random perturbation is an effective geomasking technique, to some extent superior to affine and aggregation masks. Kwan et al (2004) have assessed the spatial masks discussed in Armstrong et al. (1999), particularly levels of random perturbation in relationship to disclosure risk since mapped locations of disease or crime contain wide variation in of population density. The amount of noise added to location can be allowed to vary with population density. This idea has also been discussed by VanWey et al. (2005) who simulated a sampling frame of public schools in the US. Their data contained the geographical location of each school with potentially sensitive attribute information. A solution was proposed whereby map symbol size was adjusted to cover multiple schools, providing locational uncertainty in proportion to a specified level of identification risk. For schools in large cities (densely populated) a much smaller point buffer was needed than in remote rural areas.

In this paper we propose a geographically intelligent method of statistical disclosure control for aggregate census data which draws on elements of these geoprivacy approaches by protecting the locations of individual records. Although described in the census context, the method would be applicable to administrative or survey data. In the following section we review the disclosure control problem. In section 3 we consider traditional record swapping methods which are essentially aspatial and propose a local density swapping approach which takes into account the distribution of population as a spatial indicator of the risk of disclosure. Furthermore, we examine ways of offering greater protection against differencing by perturbing larger samples of the data but adding smaller amounts of noise. 
The remainder of the paper then considers an empirical application of the record swapping approaches. Section 4 describes the creation of a census-like microdataset; section 5 presents some measures of risk and utility and section 6 presents the results of our analysis. The results of our experiments are discussed in section 7 before drawing conclusions identifying further research priorities.

\section{Statistical Disclosure Control}

\subsection{Census Tables}

We here outline the production of aggregate census data, drawing specifically on UK practice but with international applicability. A census collects data on attributes (e.g. age or household size) for individuals and households. The objective is usually to collect data from the entire population, although in reality some will be missed. For the purposes of this discussion we will disregard the many practical challenges of enumeration which in various ways affect data quality and consider the creation of data for publication from the database of census responses.

Tables of counts are produced by cross-classification of subsets of attribute variables. Counts will typically be for either individuals or households and, for generality, we use the term unit to denote the individual, household or other set of individuals upon which the counts are based. Geographical coordinates $(X, Y)$ are associated with each unit, typically by matching to a master address list. The data available for tabulation are compiled into a microdata file, represented by an $N \times(K+2)$ matrix, Z, where $\mathrm{N}$ is the total number of units and the rows of the matrix contain a $1 \times K$ vector $\mathrm{A}$ of values of $\mathrm{K}$ attribute variables 
International Journal of Geographical Information Science Submission

as well as values of $(X, Y)$ for the different units. We refer to the $\mathrm{N}$ units as the population.

Tables for publication are formed by specifying output zones and attribute categories and counting the number of units that fall into each unique combination of output zone and category. For example, Table 1 provides a fictitious illustration of a cross-classification of religion by long term illness (both variables in the 2001 census in the United Kingdom) for 5293 individuals in a given zone. Such tables represent spatial aggregations of the microdata file.

Table 1: Fictitious Census table: Religion by long term illness for Zone $\mathrm{H}$

\begin{tabular}{|l|l|l|}
\hline Religion & Without long term illness & With long term illness \\
\hline Christian & 3251 & 1004 \\
\hline Buddhist & 8 & 0 \\
\hline Hindu & 0 & 2 \\
\hline Jewish & 4 & 1 \\
\hline Muslim & 0 & 1 \\
\hline Sikh & 0 & 0 \\
\hline Any other religion & 13 & 3 \\
\hline No religion & 556 & 86 \\
\hline Religion not stated & 248 & 106 \\
\hline Total & 4088 & 1205 \\
\hline
\end{tabular}

\subsection{The Disclosure Problem}

We are concerned with the disclosure risk which may arise from the publication of multiple frequency tables of the type described in the previous section. There are various ways of defining disclosure for tabular outputs (Willenborg and de Waal, 2001). Most commentators suppose the existence of an intruder who has access to the published tables and attempts to use these to disclose information about the units in the population. One 
basic notion is that of identity disclosure or identification, which arises if it is possible for the intruder to establish a one-to-one correspondence between an element of a table and a known unit in the population (Bethlehem et al., 1990). Such a possibility is of particular concern to census agencies, as it would contradict the confidentiality undertakings made to respondents. For illustration, Table 1 reveals that in output zone $\mathrm{H}$ there is only one Muslim. If, via another source of information, the intruder knows the name and address of a Muslim who lives in zone $\mathrm{H}$ then they can establish a correspondence between this individual and an element in the table. Thus, identity disclosure would have taken place. Identity disclosure may occur more readily for cells with counts of one, termed cell uniques, and we treat these as risky.

It might be argued that such identity disclosure is not serious since the intruder does not gain any new information about the respondent. However, identity disclosure can be associated with attribute disclosure, which arises if the intruder can learn additional information about a unit from the published output. For example, the intruder who knows a Muslim living in zone $\mathrm{H}$ can learn from Table 1 that this individual suffers from a limiting long term illness. Identity disclosure is not, however, a necessary condition for attribute disclosure. For example, an intruder who knows someone in output zone $\mathrm{H}$ whose religion is Hindu can learn that they must have a long term illness, despite there being two such people and thus identification has not taken place. Despite such considerations, we shall in this paper focus on the risk arising from identity disclosure. Focusing on cell uniques also gives us an overall indicator of disclosure risk (since the more ones there are, the more small cell counts there are likely to be). 
International Journal of Geographical Information Science Submission

Tables produced for non-coterminous geographies may be independently safe, but when published together, can sometimes be subtracted or differenced to reveal sensitive information relating to a geographical 'sliver'. This has been termed the geographical differencing problem (Duke-Williams and Rees, 1998) and occurs when one or more output zones nest entirely within another as in Figure 1.

Figure 1: Geographical Differencing Problem: Output zones which nest within one another

(a)

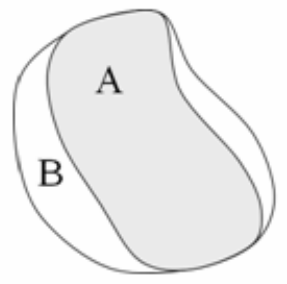

(c)

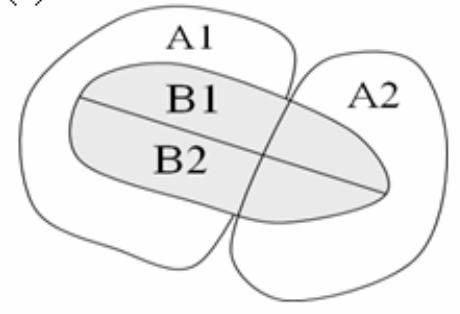

(b)

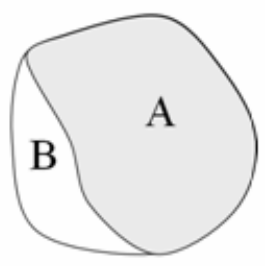

(d)

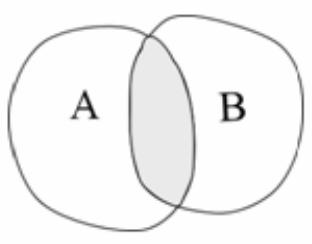

Suppose Table 2 relates to output zone B in diagram (a) or (b) in Figure 1 and Table 3 relates to output zone A, then subtracting the corresponding cells (Table 3 from Table 2) reveals a new Table 4 which relates to the differenced zone. All the non-zero cell counts are cell uniques. The geographical differencing scenario can be extended to consider output zones which when aggregated can, in combination, be differenced from a larger aggregate: for example, in diagram Figure 1(c), B2 + B1 can be differenced from A1 + A2. Sections of geographical zones which overlap but are not wholly contained are not disclosive as there is 
International Journal of Geographical Information Science Submission

no way of knowing which data units in the two tables belong to the intersecting area, as in Figure 1(d). These examples demonstrate the problems of publishing tables relating for multiple systems of output zones. Small slivers can result producing many small cell counts which are potentially disclosive. This can occur through both nested and non-nested geographies. Providing user access to an interactive tabulation tool would present many differencing problems.

Table 2: Illustrating the Geographical Differencing Problem - Fictitious Table representing Geography A

\begin{tabular}{|l|l|l|l|l|}
\hline & $16-20$ & $21-30$ & $31-40$ & $\ldots$ \\
\hline Benefit claimed & 10 & 16 & 19 & $\ldots$ \\
\hline Benefit not claimed & 8 & 12 & 11 & $\ldots$ \\
\hline
\end{tabular}

Table 3: Illustrating the Geographical Differencing Problem - Fictitious Table representing Geography B

\begin{tabular}{|l|l|l|l|l|}
\hline & $16-20$ & $21-30$ & $31-40$ & $\ldots$ \\
\hline Benefit claimed & 9 & 16 & 19 & $\ldots$ \\
\hline Benefit not claimed & 8 & 11 & 11 & $\ldots$ \\
\hline
\end{tabular}

Table 4: Illustrating the Geographical Differencing Problem - Fictitious Table representing Differenced Area

\begin{tabular}{|l|l|l|l|l|}
\hline & $16-20$ & $21-30$ & $31-40$ & $\cdots$ \\
\hline Benefit claimed & 1 & 0 & 0 & $\cdots$ \\
\hline Benefit not claimed & 0 & 1 & 0 & $\cdots$ \\
\hline
\end{tabular}

\subsection{Statistical Disclosure Control (SDC) Methods for Tabular Outputs}

A variety of SDC methods have been proposed to protect against the kinds of disclosure risk described in the previous section. They may be divided into pre-tabular methods, which are applied to microdata before aggregation into tables, for example by modifying the values of the attribute variables and post-tabular methods, which are applied to the tables, for example by rounding all cell counts to multiples of some base number, such as five (Shlomo, 2006). From a geographical perspective, pre-tabular methods are zone- 
independent, assuming that they only involve modification of the microdata file, $\mathrm{Z}$ (see section 2.1) and that standard spatial aggregation is used to form the tables from the modified microdata. If the base microdata are 'safe' then any aggregation must also be safe. Pre-tabular methods are generally more flexible, with parameters that can be varied to achieve a balance between disclosure risk and utility. Utility refers to the quality of the output after SDC and can be assessed by analyzing the impact of SDC methods on statistical analysis (Shlomo, 2006). Pre-tabular methods can cause statistical damage to the resulting tables that is difficult for the users of these tables to measure. Hence, the parameters of these methods are often set in a way to minimize this damaging effect (Shlomo, 2005). Post-tabular methods are zone-dependent since they must be applied afresh to every new table that is produced for a given output zone. Not only must any risk assessment be updated for each tabulation, but it is in theory necessary to assess that the new table cannot be combined with previously released tables to undo the protection that was previously applied. Post-tabular methods can be cumbersome to apply, particularly for very large tables. Current solutions involving rounding techniques (Salazar et al, 2004) are still in development for very large tables with a complex hierarchy of subtotals to ensure that marginal totals add up correctly whilst maintaining a high quality table. The fact that the pre-tabular SDC methods only need to be applied once is an important advantage compared to post-tabular methods, when the aim is to produce tables for multiple geographies. The remainder of this paper focuses on pre-tabular methods. In reality, the choice between these approaches is not straightforward and a combination of pre- and posttabular methods have often been implemented. Particular attention will be given here to the 
International Journal of Geographical Information Science Submission

development of a method which balances risk and utility, avoiding the need for further post-tabular adjustment.

\section{The Proposed SDC Approach}

Pre-tabular methods of SDC could involve modification of either attribute variables or geographical coordinates. The former has the major disadvantage that it can result in the introduction of inconsistencies between the attribute variables, such as a married 10-yearold. This type of anomaly will not occur as a result of modification of the geographical coordinates since most census attributes will be consistent with any geographical location. We therefore consider here only geographical perturbation methods, defined as those SDC methods which modify the true spatial locations of some or all of the units in the microdata. Thus, the coordinates $\left(X_{i}, Y_{i}\right)$ of a unit $i$ are modified to new coordinates $\left(X_{i}^{\prime}, Y_{i}^{\prime}\right)$. We refer to the distance between the old and new locations as the perturbation distance. Usually the perturbation distance, $d$ is measured in Euclidean space:

$d=\sqrt{\left(X_{i}^{\prime}-X_{i}\right)^{2}+\left(Y_{i}^{\prime}-Y_{i}\right)^{2}}$

Geographical perturbation methods may be expected to reduce disclosure risk in the aggregated table since it will not be known whether an observed cell unique in a table corresponds to a unit which genuinely falls in that cell or indeed whether the true cell count is one. Geographical perturbation could be implemented in a number of ways. One approach, applied in the geoprivacy literature to point data is that of displacement, where the coordinates of each unit are displaced according to some deterministic or random 
International Journal of Geographical Information Science Submission

procedure (Armstrong et al., 1999). One problem with this approach is that it cannot be guaranteed that the displaced locations will be feasible, for example they might fall in a self-evidently unpopulated area such as a lake. An alternative which overcomes this problem and avoids the need for detailed validation of new locations is record swapping, where pairs of units are selected from the population and the coordinates of each pair are swapped while the global set of locations is unchanged. We will focus on record swapping approaches, firstly considering in section 3.1 an established method which has been employed in practice by several census agencies (Shlomo, 2005 and Zayatz, 2006). Our proposed new approach is introduced in section 3.2.

\subsection{Random Record Swapping (RRS)}

Random record swapping (RRS) is a pre-tabular method of geographical perturbation resulting in the geographical variables of two units $i$ and $j$ being swapped. Thus, the geographical coordinates $\left(X_{i}, Y_{i}\right)$ are exchanged with $\left(X_{j}, Y_{j}\right)$ but the attribute variables remain unchanged. Different approaches may be adopted for deciding which pairs of units are swapped. Often, pairs of units are matched in some way to limit the potential damage to resulting analyses. Following Willenborg and de Waal (2001), the $i^{\text {th }}$ record of the microdata may be partitioned into three sub-vectors: $M_{i}, A_{i}^{*}$ and $\left(X_{i}, Y_{i}\right)$, where $M_{i}$ is a vector of match variables, defining a subset of the attribute variables to be controlled when seeking recipient households for swapping, and $A_{i}^{*}$ is a vector containing the remaining attribute variables. Thus, only records $j$ with $M_{j}=M_{i}$ are considered for swapping with record $i$. 
Three features of RRS can be modified, each of which may affect both disclosure risk and utility. First, the smaller the fraction of records selected for swapping, the lower both the expected risk reduction and distortion. Second, the more variables and categories which are used to match pairs of values the less distortion, but this may lead to difficulties in finding matching records for all recipients. Third, swapping can be targeted on records deemed most 'risky', determined by prior analysis of the small counts in tables (Zayatz, 2006). The UK 2001 censuses were disclosure-protected by RRS in combination with a post-tabular SDC method (Shlomo, 2006). The 2000 US census employed targeted swapping (Zayatz, 2003) in combination with post-tabular methods. The specific details of record swapping, such as the proportion of records swapped, are kept confidential so as not to aid an intruder.

To illustrate RRS further, we present the basic approach adopted in England and Wales in 2001. The units swapped were households. Match variables consisted of a hard-to-count index ${ }^{1}$, household size, and a broad age and sex distribution of individuals within the household (Shlomo, 2005). A sample of households was swapped between Output Areas (OAs: small area building brick for release of 2001 census data) within Local Authority Districts (LADs: larger area containing around 50,000 people) (Boyd and Vickers, 1999), that is each record in the sample was paired with a matching record from a different OA within the same LAD. Thus, LAD is effectively a match variable also and this method is zone-dependent, being based on specific LADs and OAs. A key drawback of such RRS is that membership of the same LAD is the only control over the distance and direction of

\footnotetext{
${ }^{1}$ Hard-to-count index was constructed from census variables known to be associated with under-enumeration such as multi-occupancy dwellings.
} 
International Journal of Geographical Information Science Submission

perturbation, which may result in significant damage to statistical analyses involving spatial elements finer than LADs.

\subsection{Local Density Swapping (LDS)}

The proposed local density swapping (LDS) method is designed to overcome this key drawback, by applying pre-tabular perturbation to the microdata in a way which is not dependent upon the choice of output zones. The first feature of the method is that perturbation distances are sampled from a probability distribution. This allows greater control because the type of distribution can be selected, as can its parameters such as mean and standard deviation.

The risk of identifying an individual by differencing depends on the proximity of other individuals with similar characteristics. For example, an elderly man located in an area with mainly young people will present a high disclosure risk. In general, the more people there are in the area, the more likely it is that someone else will share the same characteristics following Tobler's first law of geography (Tobler, 1970) similar characteristics are most likely to be found together. Thus a fundamental predictor of disclosure risk is population density. In a densely populated urban area, less perturbation is generally needed to disguise the true location of a household. A similar concept was identified earlier in the geoprivacy literature (Armstrong et al. 1999, VanWey et al, 2005), masking locations of disease or crime on a map in proportion to population density. Density is not taken into account by conventional approaches to record swapping. Thus in a densely populated region, more perturbation than necessary may be added to a household. Our second key proposal is to take account of population density by defining perturbation distance as household distance 
International Journal of Geographical Information Science Submission

as opposed to Euclidean distance. The household distance $n$ is a measure of the number of households between swapped households.

Our third proposal aims to reduce the risk from geographical differencing by swapping more records but by smaller distances. Thus a cell unique in a differenced area should have a likelihood of having been perturbed. Perturbation at the local level will minimise damage but introduce uncertainty. In practice this could be implemented by swapping broadly at the same scale as the small area geography (for the UK this might be adjacent postcodes for example, or blocks in the US) with variation depending on population density. Postcodes or blocks represent local areas for which an intruder might know detailed information about their neighbours and around which we might want to add noise. The initial sample of records selected for swapping could be random within strata (as in the random record swap) to ensure even protection against risk.

One advantage of LDS is its flexibility. Statistical organizations differ in their assessment of tolerable levels of risk and utility. LDS parameters can be varied to achieve an appropriate balance between risk and utility. Parameters that can be varied include the sampling fraction, the probability distribution, the sample size and selection and match variables. Very rare records with unusual combinations of characteristics (such as a 16 year-old-widow) are unlikely to be protected by swapping (as they tend to be unique irrespective of geography) but these are likely to require separate recoding or suppression regardless of the general protection methods applied. 


\section{Creating a Test Dataset}

It is not possible to use an actual census database due to the census confidentiality constraints. However, anonymised microdata samples and small area aggregations are available from the UK censuses (Dale and Teague 2002, Dale and Marsh, 1993). In this section we briefly describe the creation of a synthetic microdataset created from these sources to create a $100 \%$ dataset consisting of the full set of census variables, to which we assign point locations. The aim is to create a dataset that is realistic for testing disclosure control methodologies rather than to accurately replicate a specific regional population.

\subsection{Synthetic Population}

Our synthetic population is based on 1991 UK census microdata, as 2001 files were not released until after the commencement of this work. We use the Household Sample of Anonymised Records (SAR), a microdata file for a $1 \%$ sample of individuals in households, including the complete set of census variables but with geographical coding limited to Standard Region. We also use Small Area Statistics (SAS), which give information on individuals and households in tabular form at enumeration district (ED) level, the smallest areas in the 1991 census geography. EDs are similar in size to OAs containing around 200 households or 450 people. SAS tables contain the entire population for each small area. However the table dimension is small, containing only two or three variables, so the joint distribution for the full set of census variables is not known. Microsimulation techniques are used to estimate this joint distribution at the small area level. Our aim is to populate census small areas with 'plausible' households and 
International Journal of Geographical Information Science Submission

individuals drawn from the microdata sample, broadly preserving actual marginal characteristics.

Since swapping involves distorting the relationships between geography and attribute data, it is important to study an area which is spatially heterogeneous. The county of Hampshire in Southern England was chosen because of its diverse characteristics. It includes two densely populated urban areas (Portsmouth and Southampton) and extensive rural areas. The population of approximately 1.5 million displays wide variety of economic activity, household structure and individual characteristics. The county comprises 3175 EDs in thirteen LADs with population densities ranging from 138 people per $\mathrm{km}^{2}$ in Winchester to over 4000 people per $\mathrm{km}^{2}$ in Portsmouth.

A microdataset with a realistic spatial distribution is needed in order to investigate the effects of locational swapping. There are various techniques available including iterative proportional fitting based on a set of small area tables (Williamson et al., 1998) or synthetic probabilistic reconstruction (Birkin and Clarke, 1989), but here we use a method of combinatorial optimisation developed by Williamson and Voas (2000). This approach uses the SAR as the parent population from which households can be drawn to recreate populations for individual EDs. This is performed iteratively by selecting a combination of households from the SAR that best reproduces the characteristics of the ED. Constraints on the combination of households chosen are provided by known tabulations of ED data from the SAS. We have chosen four small area tables from the 1991 small area statistics covering age, marital status and sex; household space type and tenure of households; primary economic position and age; and tenure and socio-economic group of the head of 
household. The more tables used as constraints, the more iterations are required to give a good level of fit.

Figure 2 gives an overview of the microsimulation process. The first step is selection of an initial sample of households for each ED. Rather than sampling at random, the ONSCLASS variable (Wallace et al, 1995) was used to stratify the SAR records. ONSCLASS is a census-based classification of wards present on both the SAR and SAS. This is a very useful variable since it provides a key to the characteristics of the ward in which the household is located. An initial sample was taken at random from the stratum representing the same ONSCLASS as the ED. Communal establishments were excluded from our analysis. At each iteration a new household is sampled from the appropriate stratum and if the fit is improved the new household kept, otherwise it is dropped. This iterative process continues until a satisfactory fit is achieved. To measure fit we compared the simulated table frequencies, denoted $F_{c}$, to the constraint table frequencies, denoted $O_{c}$, (Ballas et al. 1999, Williamson and Voas, 2000). The subscript $c$ denotes a cell in the table for which the constraint frequencies are available. We used a measure called Total Absolute Error less than 3, denoted TAE and defined by:

$\mathrm{TAE}=\sum \max \left[\left|O_{c}-F_{c}\right|-3, \quad 0\right]$

This fit measure ignores absolute errors $\left|O_{c}-F_{c}\right|$ of less than 3 in order to speed up the process since an exact fit was not required. 
Figure 2: An overview of the microsimulation process

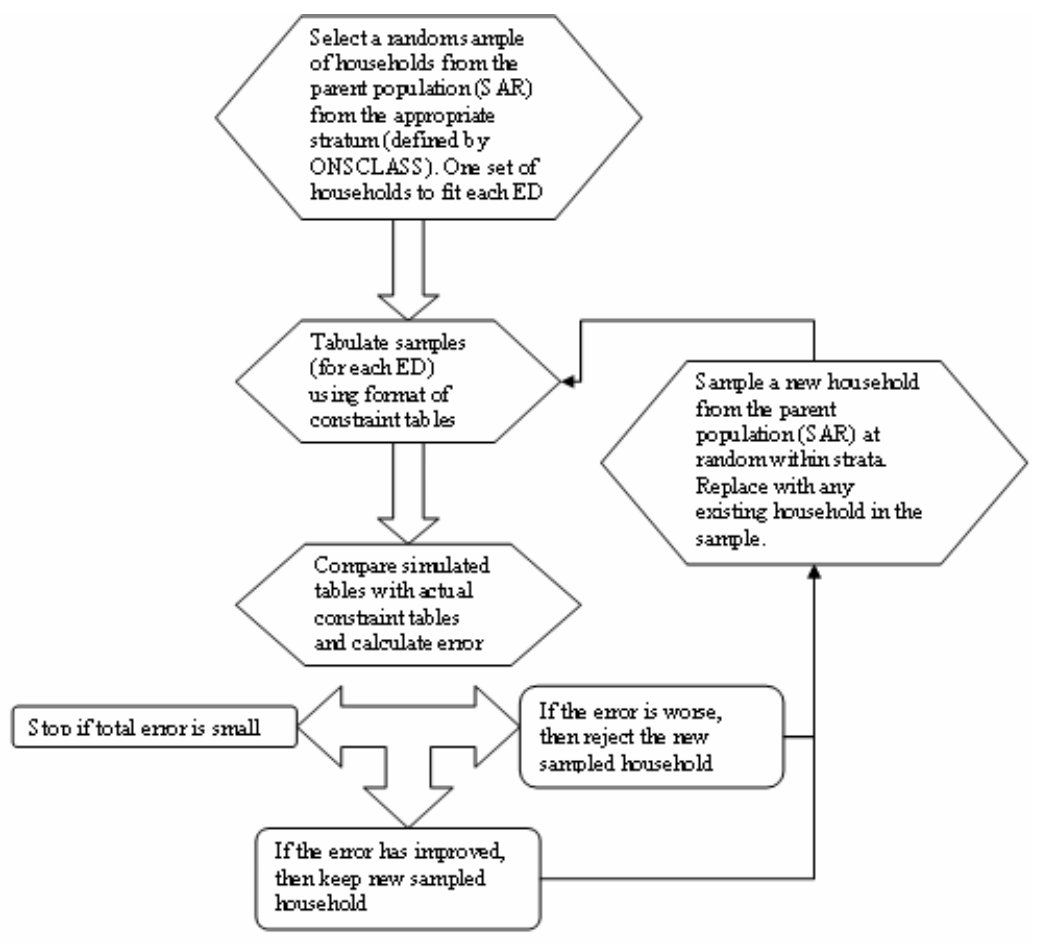

The resulting synthetic population provided an adequate fit to the published tables using this approach. There were no clusters of error which may have raised concerns that an area with unusual characteristics had not been fitted properly. Urban areas showed the worst fit but overall the TAE was relatively small at approximately 2 to 4 individuals or households per cell. $70 \%$ of households were not unique, with $15 \%$ of households sampled three times (representing $45 \%$ altogether) but less than $1 \%$ represented more than four times. This would be a problem if the objective involved assessing uniques in the entire microdataset since only $30 \%$ are unique records. However there are many uniques at the small area level in tables of two to four variables. Moreover the majority of repeated households are located in different LADs. This is an important consideration because households could be swapped which are identical thus biasing the empirical results (utility will appear to be 
better on the synthetic population than on a real population). An additional consideration is that both the LDS and RRS tests will be equally affected by any biases in the synthetic population.

\subsection{Creating Spatial Locations}

Creating spatial locations for the households is particularly important because these will be used by the swapping procedure. There are no files directly recording household locations in the UK. Instead, the directory of postcodes and enumeration districts was used, which provides a household count for each ED-postcode intersection and grid references for each postcode (Martin, 1992). Unique locations were created for households by adding noise around postcode locations, proportional to ED population density. An incomplete sort of households by tenure type was performed before grid reference allocation, resulting in households of similar tenure displaying some spatial clustering.

Figure 3: Creating artificial household locations

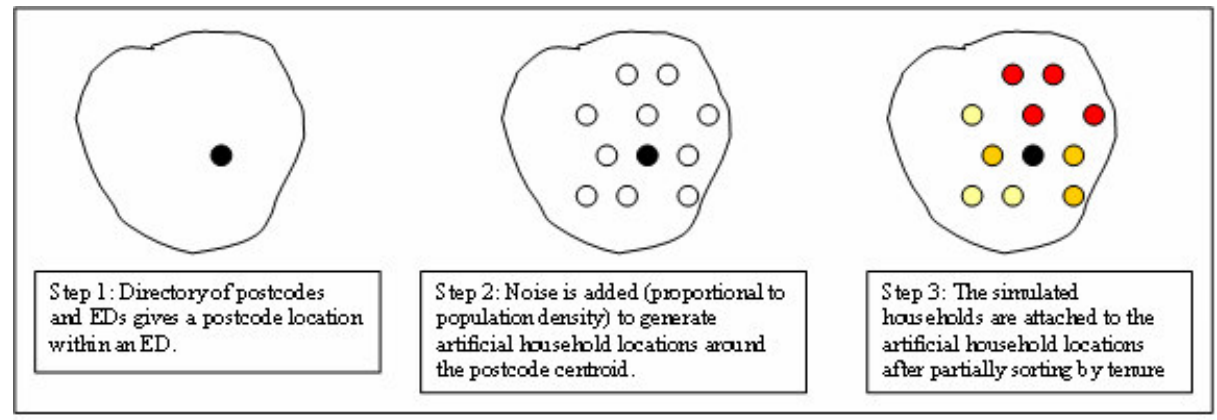

The smallest postcodes do not have formally defined boundaries, so these were generated as Thiessen polygons around postcode centroids (DeBerg et al, 2000), and adjacent 
International Journal of Geographical Information Science Submission

postcodes identified, using the deldir ${ }^{2}$ package in the statistical package $\mathrm{R}$. We thus create a synthetic micropopulation for Hampshire consisting of individuals within households having a full set of census variables. Each household has a unique point location and is assigned to a postcode, ED and ward. The postcodes have synthetic boundaries but do not necessarily nest entirely within EDs; EDs nest entirely within wards and wards within LADs.

\section{Implementing and Evaluating the Swapping Methodology with the Test Dataset}

This section describes the implementation of the general methodology proposed in Section 3 using the statistical program SAS and the synthetic population and presents measures for the assessment of risk and utility. We refer to the synthetic data as the 'original' (unswapped) data.

\subsection{Swapping Methodology}

RRS and LDS are performed by selecting an initial sample of households from the population and then finding matching households with which these will be swapped (section 3). Match variables of ethnic group, family type, number of persons in household and tenure are used. These were chosen as being similar to those used in the actual 2001 UK census RRS. RRS requires matching households in a different ED but within the same LAD.

To implement LDS, a 'distance', $n$, is generated randomly from an exponential distribution with a specified mean $\lambda$ for each of the households in the initial sample. This

\footnotetext{
${ }^{2}$ Information on the deldir package in $\mathrm{R}$ can be found at: http://rweb.stat.umn.edu/R/library/deldir/html/00Index.html
} 
distance will determine the number of households, $n$, in the circle for which the initial household is at the centre and the matching household is on the circumference. For ease of computation, households are assigned to a $100 \mathrm{~m}$ raster and a cellular approximation to a circular search performed. The circular band corresponding to number of households $n$, is then determined by counting the households in successive bands until the cumulative count is greater or equal to $n$. The outer band of households contains the $n$th household.

Generating $n$ from an exponential distribution ensures $n$ cannot be negative and has a rapidly decreasing probability of taking a large value. The probability density function of $n$ is given by:

$$
f(n)=\lambda^{-1} e^{-n / \lambda}, \quad n \geq 0
$$

so that $\lambda=E(n)$. In the following experiments a value of $\lambda$ has been specified which represents an average distance between adjacent postcodes. A random swap of $10 \%$ of households between adjacent postcodes was performed and $\lambda$ was determined as the mean number of households between all pairs of swapped households. The minimum and maximum from this $10 \%$ random postcode swap was also used to truncate the distribution to prevent swapping over very long distances or very small distances.

Once the circular band containing the $n$th household is determined, a best matching household from the households in this band can be found using the match variables of ethnic group, tenure, persons in household, family type. Households which have previously been swapped are penalized and discouraged from swapping a second time.

\subsection{Evaluating Effectiveness in a Risk-Utility Framework}


Following Duncan et al (2001), we propose to evaluate the effectiveness of the swapping methods in a risk-utility framework, i.e. we study the performance of each of the methods in terms of both disclosure risk and the utility of the resulting outputs for analysis. Moreover, since the methods depend upon the specification of parameters, such as the proportion of records to swap, we shall study how such choices affect risk and utility and the trade-off between the two. In order to set up this framework we need to introduce measures of risk and utility.

\subsubsection{An Indicator of Disclosure Risk Disclosure protection can be measured by} comparing disclosure risk before and after perturbation. In section 2, we discussed how risk before perturbation could be measured in terms of cell uniques. A crude measure would be the number of cell uniques. After perturbation, cell uniques may also be considered, since these might be a natural target for an intruder, but it would be inappropriate to still measure risk by the number of cell uniques, since these may no longer be genuine. Instead, the probability that an observed cell unique (after perturbation) represents an actual unique is considered.

Such measures of risk are clearly dependent upon output zones. These may be EDs or wards, for example. To measure the risk arising from geographical differencing, the disclosure risk for frequency tables for zones assumed to be equivalent in scale to a differenced 'sliver' is considered, for which the smallest zones available are postcodes (and are independent of the geographies used in the methodology).

The cells in a table $T$ are defined by cross classifying a subset of the attribute variables in the vector $A$ (see section 2.1). An arbitrary cell $c$ in this table is defined by a combination of 
International Journal of Geographical Information Science Submission

the categories of this subset of attribute variables. Let $F_{c}$ denote the cell frequency in a specified zone, that is the number of units in the zone with the specified combination of values of the attribute variables. To be explicit about the effect of the perturbation process, let $F_{c}^{o}$ denote the cell frequency before perturbation and $F_{c}^{p}$ the cell frequency after perturbation. Also let $n_{T}$ denote the number of cells in table $T$ and let match $=1$ if $F_{c}^{o}=F_{c}^{p}=1$ and if the same unique household appears in the table before and after perturbation. A cell count of one after perturbation is called a true unique if match $=1$, i.e. if it was also a count of one in the original table and it relates to the same household. The probability of finding a true unique is thus:

$\operatorname{Pr}(T U)=\frac{\sum^{n_{T}} I\left(F_{c}^{o}=F_{c}^{p}=1 \& \text { match }=1\right)}{\sum^{n_{T}} I\left(F_{c}^{p}=1\right)}$

where the sums are over all the cells $c$ in the table and $I$ is an indicator function which equal 1 if true, 0 otherwise.

5.2.2 Measures of Utility Utility will be measured in terms of distortion to the data, i.e. in terms of dis-utility. Specifically, we measure the absolute average deviation per cell (AAD) averaged across all tables at a particular level of geography. AAD will be measured on tables formed by cross-classifying age, sex and marital status. These variables were chosen as they contain vital demographic information which should not be distorted and since they do not include any of the matching variables. The measure is defined as:

$$
\mathrm{AAD}=\frac{\sum^{n_{T}}\left|F_{c}^{p}-F_{c}^{o}\right|}{n_{T}}
$$


International Journal of Geographical Information Science Submission

Section 6.5 considers some additional measures of utility, which assess how swapping distorts spatial features of the tables.

\section{Results of Applying Swapping Methodology to Test Dataset}

\subsection{Comparison of $10 \%$ RRS with $10 \% \mathrm{LDS}$}

We begin our analysis by simulating a $10 \%$ RRS, taken to be a realistic option which might be employed in a census, and compare with a $10 \%$ LDS. The methods are applied to the entire synthetic population of Hampshire. The same initial 5\% random sample (forming one half of the swapped records) was used in both cases, with the number of records selected proportional to the total population in each ward. This ensured an even distribution of records for swapping over the entire county.

Table 5 presents disclosure risk in terms of (a) percentage of 'true uniques' and (b) number of true uniques per 1000 population at risk. We assess disclosure risk in tables of long term illness and ethnicity since these variables are likely to produce many small cell counts. Table 5(a) shows that using matching variables (the variables $M_{i}$ (section 3.1 ) on which both households must match for a swap) as opposed to swapping households at random, the resulting disclosure risk is higher. There is very little difference between the two methods when swapping only $10 \%$ of households. More importantly the disclosure risk after applying both RRS and LDS is very high at all levels of geography at over $80 \%$. This means there is a high probability that a cell count of one relates to the original household. This is likely to be considered unacceptable by a statistical agency and in this case it would seem sensible to apply a post-tabular method to the data (as in 2001). Table 5(b) also shows that disclosure risk is highest for small zones, i.e. at postcode level with approximately 7 
International Journal of Geographical Information Science Submission

true uniques per 1000 population at risk. At higher levels of geography, there are far fewer uniques.

Table 5: Assessing Disclosure Risk (Percentage of True Uniques)

\begin{tabular}{|c|c|c|c|}
\hline \multicolumn{4}{|c|}{ (a) DISCLOSURE RISK (proportion of true uniques) } \\
\hline & Postcode & ED & Ward \\
\hline $10 \%$ Random Record & 0.94 & 0.88 & 0.81 \\
\hline \multicolumn{4}{|l|}{ Swap (with matching) } \\
\hline $10 \%$ Random Record & 1.00 & 0.93 & 0.88 \\
\hline Swap (without matching) & & & \\
\hline $10 \%$ Local Density Swap & 0.89 & 0.92 & 0.92 \\
\hline \multicolumn{4}{|c|}{\begin{tabular}{l|l|l|} 
(with matching) & \\
\end{tabular}} \\
\hline \multicolumn{4}{|c|}{ (b) DISCLOSURE RISK (number of true uniques per 1000 population at risk) } \\
\hline & Postcode & ED & Ward \\
\hline $10 \%$ Random Record & 7.55 & 3.13 & 0.30 \\
\hline \multicolumn{4}{|l|}{ Swap (with matching) } \\
\hline 10\% Local Density Swap & 6.79 & 2.92 & 0.27 \\
\hline (with matching) & & & \\
\hline
\end{tabular}

Table 6 presents the utility of the data after swapping, measured in terms of deviation between cell counts in the original and protected tables. As before, an average is taken over all tables at a particular level of geography. In general the LDS produces smaller average cell deviations.

Table 6: Assessing Utility (Absolute Average Distance)

\begin{tabular}{|l|l|l|l|}
\hline UTILITY & Postcode & ED & Ward \\
\hline 10\% Random Record Swap (non-matching) & 0.25 & 1.11 & 6.66 \\
$10 \%$ Random Record Swap (matching) & 0.17 & 1.09 & 5.75 \\
\hline $10 \%$ Local Density Swap (matching) & 0.24 & 0.97 & 4.40 \\
\hline
\end{tabular}


International Journal of Geographical Information Science Submission

\subsection{Increasing the sampling fraction}

Since the $10 \%$ swap results in high disclosure risk we next examine how the risk-utility outcome changes as we increase the total proportion of records swapped. In this scenario, we use only a sub-region of the synthetic population relating to the Basingstoke and Deane local authority because the methods are computationally intensive. Initial samples are drawn which when paired with matched households make total swapped samples of $10 \%$, $25 \%, 50 \%, 70 \%, 90 \%, 100 \%$. In this case the samples were completely random with no stratification by ward. Moreover each sample was independent meaning that the $10 \%$ RRS initial sample was different to the $10 \%$ LDS initial sample. In figure 4 we measure risk at postcode level as it is the sliver level which presents the greatest disclosure risk (see table 5). The measure used is the probability of being a true unique. However, utility is measured at ward level representing a more common scale for analytical use.

Figure 4: Comparing the Risk-Utility outcome for LDS and RRS with different sampling fractions

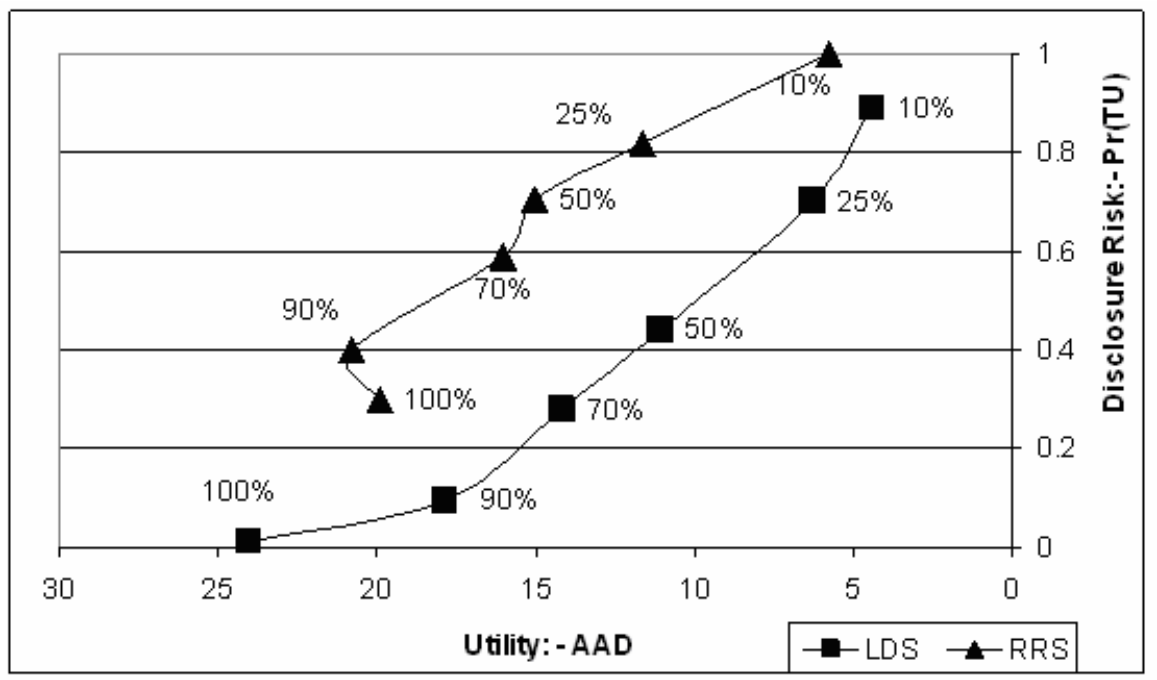


The graph shows how LDS improves upon RRS across all sampling fractions. Thus, for a given utility (a vertical line on the graph), LDS always has a lower disclosure risk at postcode level than RRS. Conversely, for a given level of risk (a horizontal line on the graph), LDS always achieves greater utility at ward level than RRS. Suppose a statistical agency wanted to ensure disclosure risk was below 0.5 ; following figure 4 , they would need to swap approximately $70 \%$ of the records to achieve this through RRS but around $50 \%$ of the records would need to be swapped if LDS was used. Moreover if 50\% of records were swapped with LDS, we would still obtain higher utility at ward level than if $70 \%$ of the records were swapped with RRS.

\subsection{Changing the Mean Perturbation Distance for LDS}

The sampling fraction is one parameter of the LDS method that can be changed. Another is the mean perturbation distance. This distance is measured in terms of number of households and thus doubling the perturbation distance does not mean the households are moved twice as far in Euclidean space. The relationship between the area of the circular band and the radius of the circle containing $n$ households is not linear and this needs to be taken into account when selecting an appropriate perturbation distance. A small sample size of $10 \%$ would require setting $\lambda \geq 10,000$ households to reduce disclosure risk by a significant amount (less than 50\%) with an average distortion of 5 per cell. On the other hand, with a sampling fraction of $70 \%$, to reduce disclosure risk below 0.5 at ward level, $\lambda=2,000$ households would be appropriate but the distortion per cell would be 15 . 


\subsection{The relationship between Geography and the $R-U$ Outcome}

In Figure 5, we show the general pattern in terms of risk-utility over different output scales. These results include a completely independent geography derived from the 2001 census: Lower Super Output Areas. LSOAs are larger than EDs but smaller than wards. Risk here is measured in terms of the probability of being a true unique for the respective geography (i.e. postcode, LSOA or ward). Utility is the AAD for the respective geography. The figure shows a definite scale effect. As the zone size increases, the utility worsens in terms of AAD, with wards having the greatest average cell deviation and postcodes having the smallest average cell deviation: the larger zones of course have larger populations. However, the most important effect observable in Figure 5 concerns the disclosure risk at postcode level. LDS results in better utility (lower AAD) and lower risk than RRS for equivalent sampling fractions $(0 \%, 10 \%, 25 \%, 50 \%, 70 \%, 90 \%, 100 \%)$ as indicated by the positioning of the lines. However, it is difficult to detect any difference between the methods at the higher levels of geography; partly because of the more unpredictable effect of RRS. Similar patterns were picked up for OAs and EDs (not much difference between the two methods) but are not included in the graph for clarity. 
Figure 5: Comparing the Risk-Utility Outcome of LDS with RRS over different levels of geography

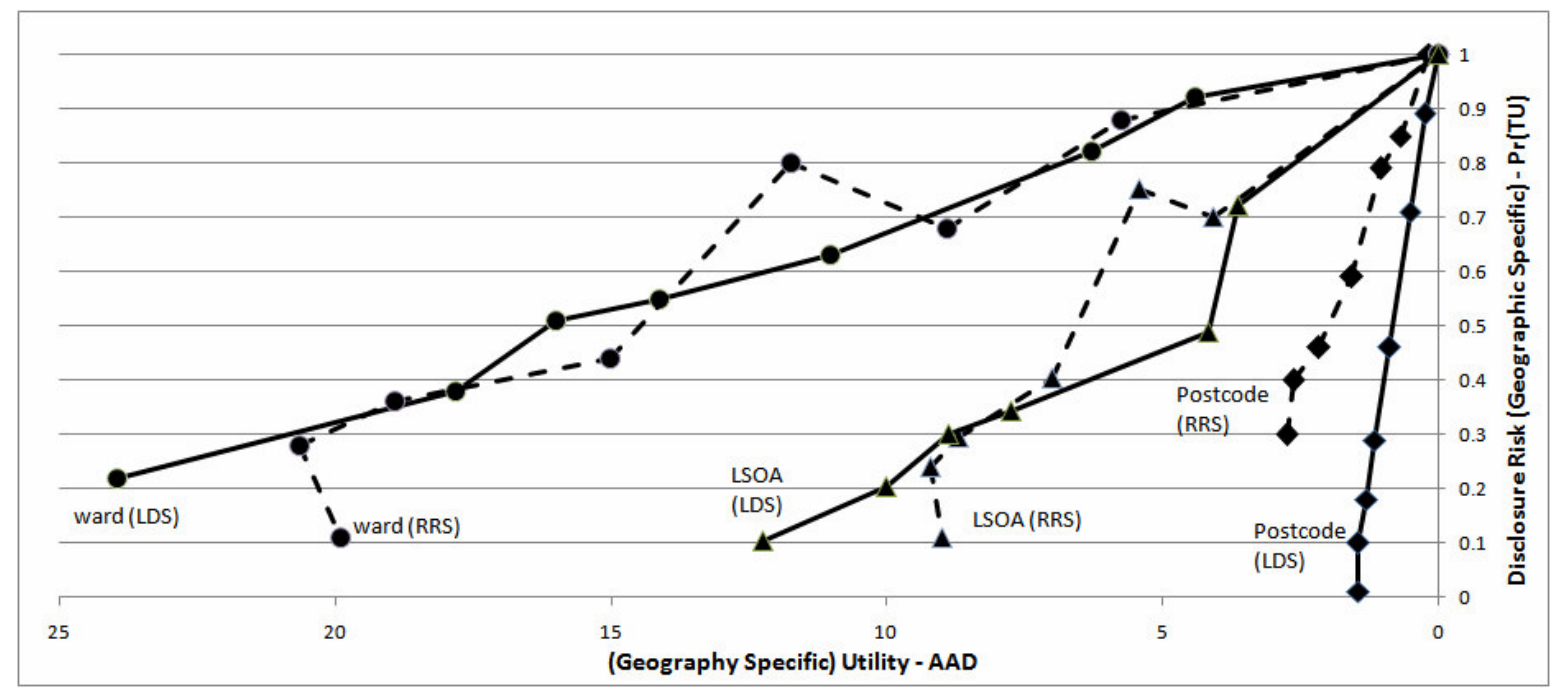

The sample sizes have been omitted from the graph for clarity; however the lines are joined in order of increasing sample size.

\subsection{Spatial Measures of Utility}

Measurement of utility in terms of AAD may have masked underlying effects not picked up by averaging. In this section we attempt to study utility in more depth. As discussed in section 3.1 , the perturbation methods swap a record $i$ with values $\left(M, A_{i}^{*}, X_{i}, Y_{i}\right)$ with a record $j$ with values $\left(M, A_{j}^{*}, X_{j}, Y_{j}\right)$ so that, after swapping, record $i$ has values $\left(M, A_{i}^{*}, X_{j}, Y_{j}\right)$. Thus, the relationship between the match variables $M$ and the attribute variables $A^{*}$ is unchanged. Similarly the relationship between the geography and match variables is unchanged. For example, we would expect the relationship between tenure and occupation to be the same after swapping. In fact this is an important advantage of geographical perturbation methods over other disclosure methods. Rounding, for 
International Journal of Geographical Information Science Submission

example, distorts the interrelationships between the attribute variables, often artificially increasing correlations (Shlomo, 2005; 2006). However, the relationship between the geography variables and the attribute variables is distorted with swapping. Thus when searching for appropriate utility measures, it would seem sensible to focus on measures in a spatial context. In this section we shall study utility primarily at LSOA level, representing a common scale for policy-making and analysis.

6.5.1 Changes in spatial rank We first consider how zones change relative to one another in terms of their ranking for a particular attribute. We are interested in changes in overall spatial pattern, such as would alter the shading classes on a choropleth map; that is changes in rank order rather than changes in scale. We here sort zones according to an attribute and assign these ranks into groups, comparing the rank group for each zone before and after swapping.

The test will be carried out on LSOAs for two different attributes: (1) percentage unemployment and (2) percentage of male head of households, aged 35-50, in a professional job with a first degree or higher. As with any large mixed urban/rural area, these attributes are likely to vary over space. The latter is a category formed from a crossclassification of the variables and will show the extent to which interactions of the variables are distorted by geography. The LSOAs are split into deciles. We present the results in Table 7 as absolute percentage change (in rank group) showing the median of the approximate normal distribution and the maximum. The table shows that, in general, LDS results in fewer rank changes than RRS. The cross-classified attribute histograms also showed similar patterns so have not been included here. This indicates that the underlying patterns in the data are likely to be more distorted by RRS than LDS. 
International Journal of Geographical Information Science Submission

Table 7: Absolute Percent Changes in Rank Group for LSOAs: Comparing LDS and RRS for 25\% and $80 \%$ swaps.

\begin{tabular}{|l|l|l|l|l|}
\hline & RRS25 & LDS25 & RRS80 & LDS80 \\
\hline Median & 2 & 1 & 2 & 2 \\
\hline Maximum no & $29 / 103$ & 9 & 9 & 20 \\
\hline $\begin{array}{l}\text { Proportion } \\
\text { change }\end{array}$ & & $36 / 103$ & $11 / 103$ & $17 / 103$ \\
\hline
\end{tabular}

6.5.2 Effect on Spatial Autocorrelation Another way of assessing changes in spatial distributions is to study the effect on clustering / spatial autocorrelation (Fotheringham et al, 2002). Swapping is likely to distort patterns of spatial autocorrelation. In particular, swapping over large distances is likely to make the data more homogeneous and pockets of households exhibiting unusual characteristics would tend to become more like the region as a whole. Therefore if we know of a variable (or set of variables) which exhibit spatial dependency, we can exploit this relationship to assess the effect of the two swapping methods.

Typically a single measure of spatial autocorrelation is calculated which describes an overall degree of spatial dependency across the whole dataset. Local measures of spatial autocorrelation allow spatial variations in the spatial arrangement of data to be examined (since a global measure may mask the true pattern). In this section we will assess spatial autocorrelation for the two attributes (a) percentage unemployed and (b) percentage of male head of households aged 35-50 in a professional job with a first degree. The results for the swapped populations will be compared against the original data.

The global measure of spatial autocorrelation to be used is the Moran's I: (Moran, 1950) 


$$
I=\frac{m \sum_{u} \sum_{v} w_{u, v}\left(Z_{u}-\bar{Z}\right)\left(Z_{v}-\bar{Z}\right)}{\left(\sum_{u} \sum_{v} w_{u, v}\right) \sum_{u}\left(Z_{u}-\bar{Z}\right)^{2}}
$$

where $m$ is the number of zones

$Z_{u}$ is the percentage in a particular category of a variable or a cross-classification of variables A, for zone $u$

$\bar{Z}$ is the mean of the percentages across all zones $w_{u v}$ is an element of a contiguity matrix, taking the value 1 if zone $u$ is a neighbour of zone $v$ and 0 otherwise.

Values of Moran's $I$ larger than 0 indicate positive spatial autocorrelation; values smaller than 0 indicate negative spatial autocorrelation.

Spatial autocorrelation at a local level will be measured using the LISA statistic (Local Indicators of Spatial Association) computed as:

$$
I_{u}=\frac{\sum_{v} w_{u v}\left(Z_{u}-\bar{Z}\right)\left(Z_{v}-\bar{Z}\right)}{\sum_{u}\left(Z_{u}-\bar{Z}\right)^{2}}
$$

Maps can be produced showing the value of $I_{u}$ for each zone $u$. In the LISA maps, highhigh and low-low relate to incidences of positive spatial autocorrelation whereas high-low and low-high relate to incidences of negative spatial autocorrelation. The Moran's I and the LISA maps were computed in $\mathrm{GeoDa}^{3}$ and relate to the Basingstoke and Deane local authority.

\footnotetext{
${ }^{3}$ GeoDa is a spatial analysis software tool developed at the Spatial Analysis Lab, University of Illinois; https://www.geoda.uiuc.edu/
} 
6.5.2.1 Spatial Autocorrelation for Percentage Unemployed The LISA maps in Figure 6 were computed for LSOAs in Basingstoke and Deane comparing the absolute change in $I_{u}$ between the original unswapped data and the swapped populations (for samples of $25 \%$ and 80\%). The original LISA map (not pictured here) showed evidence of negative spatial autocorrelation north of the centre of Basingstoke but positive spatial autocorrelation in central and southern Basingstoke. The nearest neighbour weights matrix was used with eight nearest neighbours selected. This means that there is some randomness in which 'neighbours' are selected (if there are more than eight nearest neighbours). For this reason, the same weights matrix was used in every case. Figure 6 shows that at the $80 \%$ level, there are significant amounts of absolute change in $I_{u}$ particularly in and around the central urban area. There is more change for RRS than LDS as indicated by the darker shadings of the LSOAs with absolute change being greater than one in several LSOAs. At the $25 \%$ level there were only small changes for both RRS and LDS (not displayed in this paper). All swapped populations were found to have fewer significant areas of spatial autocorrelation as the sampling fraction increases suggesting that the data, after swapping, is possibly becoming more homogeneous as might be expected. 
International Journal of Geographical Information Science Submission

Figure 6: LISA maps showing Absolute Change in $I_{i}$ between the original (unswapped) data and the swapped data, for LSOAs for \%unemployed.
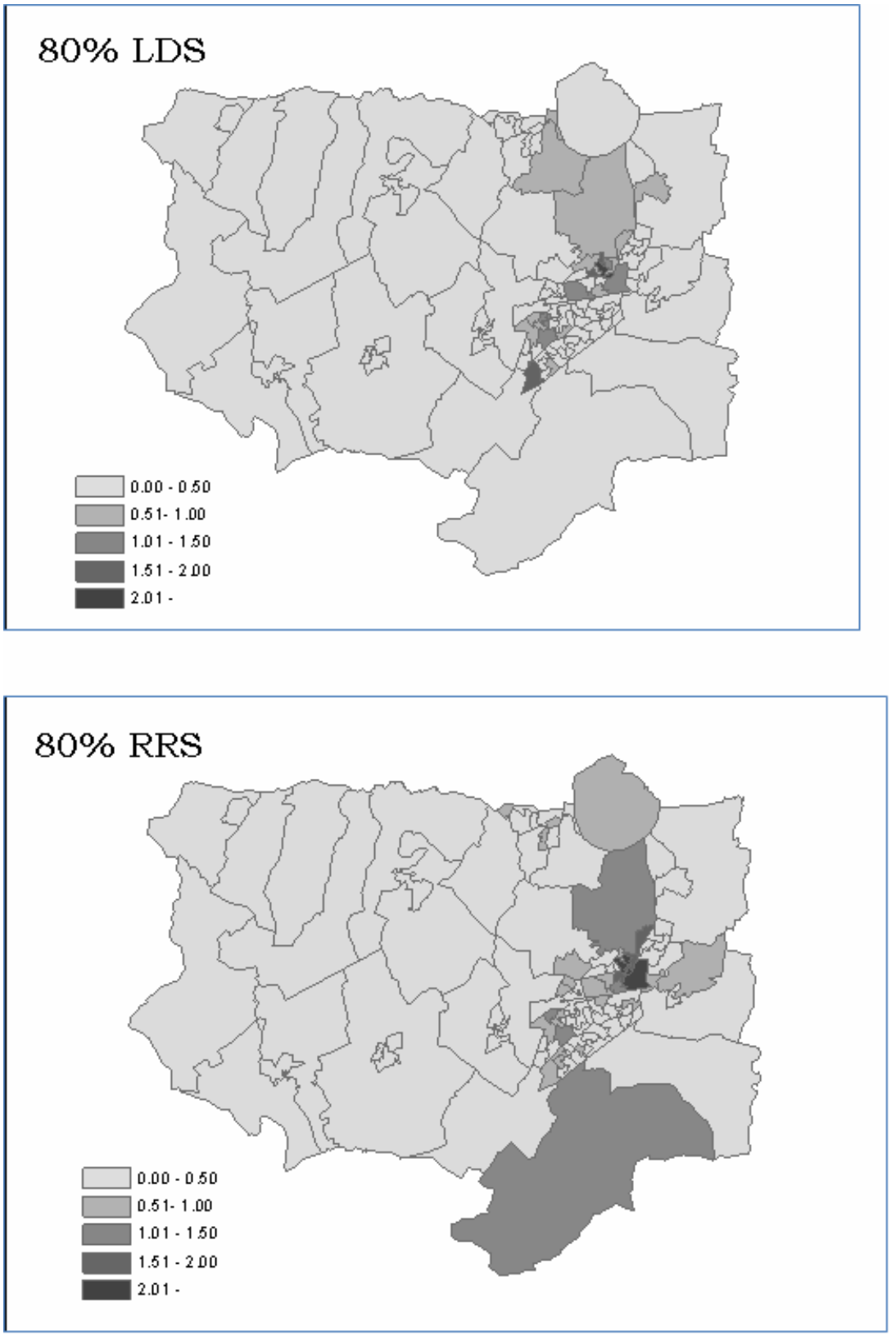

Moran's I gives a global indicator of spatial autocorrelation although it is perhaps misleading in this case with both positive and negative correlation present. Moran's $I$ is 
0.3491 for the original data and changes very little at $25 \%$ swapping. At $80 \%$ it fell to 0.2341 for RRS while remaining at 0.3338 for LDS.

\subsubsection{Spatial autocorrelation for Percentage in a Category of a Cross-Classification}

We now turn to the percentage in a category of a cross-classification of variables, represented by the percentage of male head of households, aged 35-50, with a first degree or higher in a professional job. As expected this percentage is broadly inversely related to levels of unemployment. As before, most of the structure is retained at the $25 \%$ swap rate, perhaps more so for the LDS, with little change in $I_{u}$ for the LSOAs (not shown). On the other hand at the $80 \%$ level, there is major damage to the autocorrelation structure as indicated by the dark shaded LSOAs for RRS in Figure 7. Further investigation for RRS showed that many of the significant LSOAs showed incorrect directions of spatial autocorrelation after swapping. This observation is also supported by Moran's $I$. At $25 \%$ the spatial correlation is little changed from the 0.2640 for the original data for both RRS and LDS. At $80 \%$ swapping this has reduced to 0.0367 for RRS whereas for LDS it remains at 0.2336 . RRS swaps over much larger distances, having the potential for much greater damage than LDS swapping, primarily over shorter distances. This divergence between the methods is more apparent for the cross-classification than for a single variable. 
International Journal of Geographical Information Science Submission

Figure 7: LISA maps showing Absolute Change in $I_{i}$ between the original (unswapped) data and the swapped data, for LSOAs for \% male heads in professional job aged 35-50 first degree.
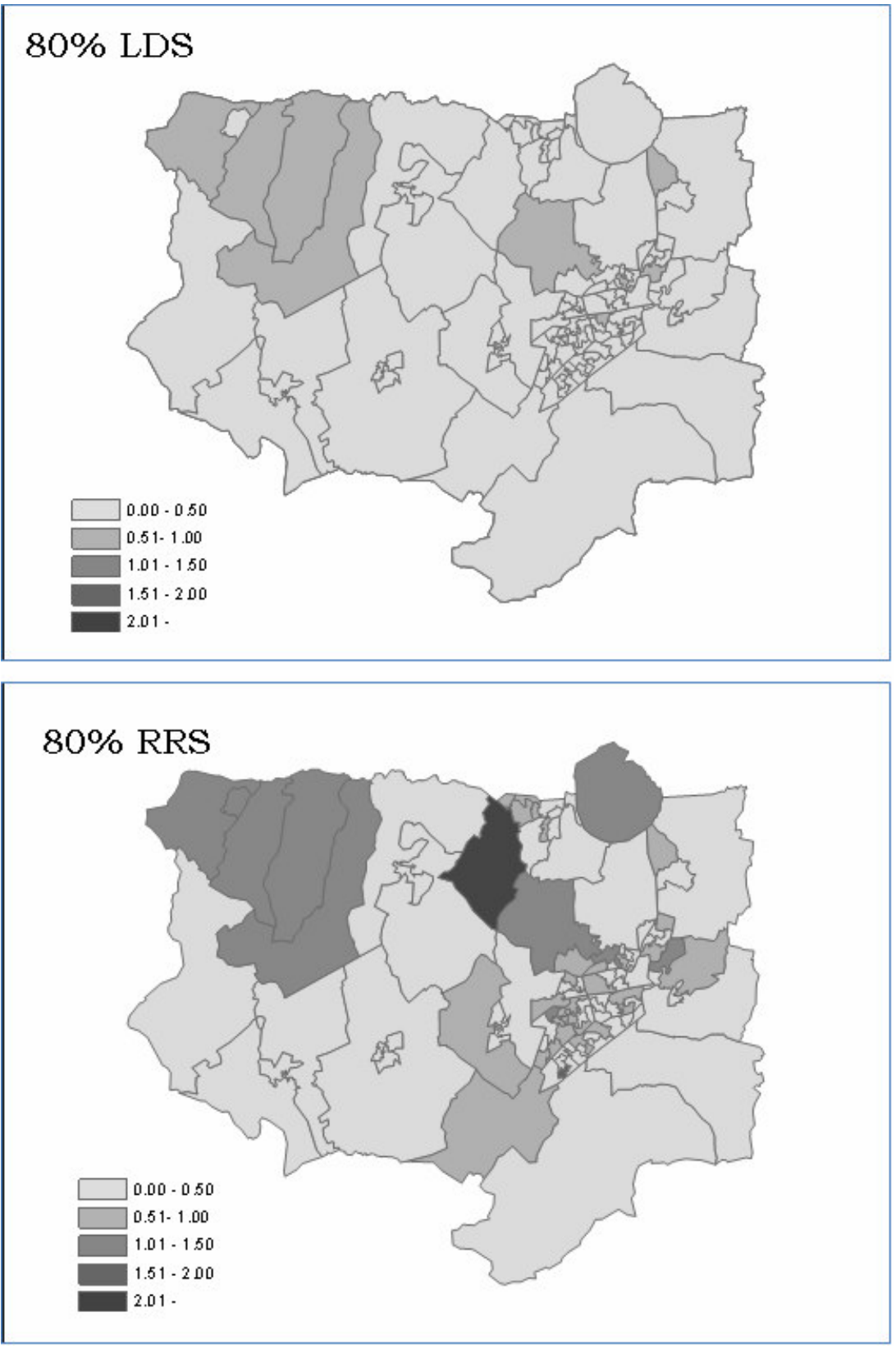

OA-level maps (not shown) display similar patterns to the LSOA maps shown here but the small size of the OAs makes visual interpretation of the patterns difficult. In conclusion, 
International Journal of Geographical Information Science Submission

the work in this section provides some evidence the LDS is less damaging to the detailed spatial data structure than RRS.

\subsection{Discussion of Results}

We here discuss some of the principal themes emerging from our results. Swapping records shorter distances and in proportion to local population density using the LDS method does seem to be effective in reducing disclosure risk at the postcode level and has the important benefit that it permits small area tables to be produced with less risk and provides stronger protection against geographical differencing. Although more noise is added at the local level, similar levels of protection and damage to those seen for more conventional methods are observed for larger zones. In addition, LDS makes no reference to pre-existing geographical boundaries and is thus more resilient to future reaggregation and differencing challenges.

We have begun to assess the utility of the new method by studying the changes in spatial relationships after swapping. The LISA maps showed that with LDS, the most significant patterns in the data still remained after a large proportion of records were swapped. However with RRS, patterns were lost at lower levels swapping, particularly for the crossclassified attribute. Changes in rank order indicated that LDS was altering the data less than RRS, which is particularly relevant to GIS and mapping applications. LDS achieved a better outcome than RRS at the postcode level, producing smaller average cell deviations in a table made up of the independent variables age, marital status and sex. Many different utility measures could be employed but in general LDS retains higher utility than RRS because households are only moved in proportion to local population density, with 
International Journal of Geographical Information Science Submission

households in high density areas being moved only short distances. In general, this contributes to the RRS results being more unpredictable than those for LDS.

A statistical organization would normally desire that disclosure risk should be less than $50 \%$ so that the odds are against an intruder finding a true unique. Ideally the disclosure risk would be even smaller than this, at around $10 \%$ or less. Swapping a $10 \%$ sample under either method was very ineffective and the percentage of true uniques remained above $80 \%$. Even swapping $25 \%$ of the data resulted in a disclosure risk still above $50 \%$ at all levels of geography and this helps to explain why utility remained so high in the LISA maps at this level. Also at the $25 \%$ swap level, the order of rankings was not disturbed too greatly. To reduce the disclosure risk below $10 \%$ for all levels of geography would probably mean swapping around $90-100 \%$ of the records under either method. Organizations would probably consider it unacceptable to implement such high swapping levels, which means it is unlikely that swapping could ever be used as a sole protection method and would probably always have to be combined with some post-tabular modification.

\section{Conclusion}

Statistical agencies wishing to provide outputs by flexible geographies need to protect against the geographical differencing problem which may arise. Pre-tabular disclosure control methods are most attractive for this purpose, because they need only be applied once, ensuring that any tables and resulting differenced areas must also be safe. In this paper, we have proposed the LDS method as an alternative to the established RRS method and have argued that it may provide greater protection at the local level as well as allowing more records to be swapped whilst retaining spatial patterns in the data. Essentially, it 
International Journal of Geographical Information Science Submission

employs greater spatial intelligence and whether or not record swapping alone is judged to provide sufficient protection, it is a powerful disclosure control method which may provide a more efficient balance of risk and utility particularly for GIS applications. A census user might only know the percentage of records swapped but this still affords a large degree of uncertainty around each cell. This contrasts with post-tabular methods such as rounding where an intruder can usually deduce a narrow uncertainty interval around each cell in relation to the rounding base. Moreover local swapping is a good way of providing confidentiality protection whilst ensuring the plausibility of the data. RRS often moves households out of their local area and thus if the two areas are very different (moved from an inner city zone to a rural zone for example), it could be obvious that a household has been swapped presenting both a disclosure risk and reduction in accuracy of the data. More work needs to done to study other measures of disclosure risk and utility. Risk assessment may, for example, be extended by looking at disclosure from zeros and other small cells and not just from cell uniques. Another idea might be to obtain two similar geographies and analyse the differencing risk attributable to the two swapping methods.

With regard to utility, most GIS use of census-type data currently ignores the impacts of disclosure control methods whereas these may in fact significantly affect geographical analysis results. Measurement of utility is far from straightforward. AAD, used here, is limited because it is an absolute value, not relative to the original cell values and since it is an average, it can mask underlying detailed effects. However AAD has provided a useful initial evaluation of the effects of changing the parameter values for swapping, due to its ease of calculation. It is important to remember that the work in this paper has been carried out for a particular synthetic population and to some extent the results will depend on the 
unique characteristics of this dataset. Since LDS is dependent on population density, it would be a logical extension to study the effects in regions of high and low density more closely. Moreover, we have focused here on record swapping, but it would also be possible to perform other types of geographical perturbation such as displacement using standard

GIS functions which are being used for geoprivacy purposes. A more comprehensive examination of utility after swapping would need to more carefully address the spatial operations that GIS census users typically apply. Much more work needs to be done, both to fully understand current practice and with regard to future census data production.

\section{Acknowledgements}

This work was funded by an ESRC postgraduate studentship: PTA-042-2004-00013.

SAR and SAS census data were taken from the Census of Population Programme web pages funded by the ESRC and additionally JISC: http://www.census.ac.uk/default.htm

\section{References}

Armstrong, M.P., Rushton, G., and Zimmerman, D.L., 1999, Geographically masking health data to preserve confidentiality. Statistics in Medicine, 18, 497-525

Ballas, D., Clarke, G. and Turton, I., 1999, Exploring microsimulation methodologies for the estimation of household attributes. In $4^{\text {th }}$ International Conference on GeoComputation, Virginia, USA, 25--28 
International Journal of Geographical Information Science Submission

Bethlehem, J.G., Keller, W.J. and Pannekoek, J., 1990, Disclosure Control of Microdata. Journal of the American Statistical Association, 85, 38-45

BIRKIN, M., and ClARKE, M., 1989, The generation of individual and household incomes at the small area level using Synthesis, Regional Studies, 23, 535-548

BOYD, M. and VICKERS, P., 1999, Record swapping - a possible disclosure control approach for the 2001 UK census. UNECE/Eurostat Work Session on Statistical Data Confidentiality, March 1999, Thessaloniki

BRown, D., 2003, Different approaches to disclosure control problems associated with geography. Joint UNECE/Eurostat work session on statistical data confidentiality, 7-9 April 2003, Luxembourg, working paper 14.

Curtis, A.J, Mills, J.W, and LeITNER, M. 2006, Spatial confidentiality and GIS: reengineering mortality locations from published maps about Hurricane Katrina. International Journal of Health Geographics, $\mathbf{5}$

DAle, A. and MARsh C, 1993, The 1991 Census User's Guide, HMSO, London.

Dale, A. and Teague, A., 2002, Microdata from the census: samples of anonymised records. In (P.ReEs, D. MARTin And P. Williamson (eds.) The Census Data System. Chichester: Wiley pp 203-212 
International Journal of Geographical Information Science Submission

DeBerg, M., Kreveld, M., Overmars, M. and SchwarzKopt, O., 2000, Computational geometry: algorithms and applications $\left(2^{\text {nd }}\right.$ ed). Springer-Verlag

Duke-WiLliams, O. and REES, P., 1997, The flexible geography task of the statistical disclosure control project: progress and thinking. First ESRC/JISC workshop planning for the 2001 Census on 'The 2001 Census - what geography do we want?' Available online at: http://www.census.ac.uk/censusdatasystem/chapter2\%5Cwp9701.pdf

Duke-Williams, O. and ReEs, P., 1998, Can Census Offices publish statistics for more than one small area geography? An analysis of the differencing problem in statistical disclosure. International Journal of Geographical Information Science, 12, 579-605

Duncan, G., Keller-McNulty, S. and Stokes, S., 2001, Disclosure Risk vs. Data Utility: the R-U Confidentiality Map. Technical Report LA-UR-01-6428, Statistical Sciences Group, Los Alamos, N.M .:Los Alamos National Laboratory.

Fotheringham, A.S. Brunsdon, C. and ChARLton M, 2002, Geographically Weighted Regression: The Analysis of Spatially Varying Relationships, New York: Wiley.

HoLT, D., 2003, The need for new statistical legislation for the United Kingdom. Journal of the Royal Statistical Society, Series A (Statistics in Society) 166, 349 - 67 
International Journal of Geographical Information Science Submission

KWAN, M.-P. and CASAS, I. and SchMiTZ, B.C., 2004, Protection of geoprivacy and accuracy of spatial information. How effective are geographical masks? Cartographica, 39, 15--28

LAMBERT, D., 1993, Measures of disclosure risk and harm. Journal of Official Statistics, 9, 313--331

LEITNER, M. and CURTIS, A., 2006, A first step towards a framework for presenting the location of confidential point data on maps - results of an empirical perceptual study. International Journal of Geographical Information Science, 20, 813-822 (10)

Martin, D., 1992, Postcodes and the 1991 Census: Issues, Problems and Prospects Transactions of the Institute of British Geographers NS 17, 350-357

MARTIN, D., 2000, Towards the geographies of the 2001 UK Census of Population. Transactions of the Institute of British Geographers, 25, 321--332

MorAN, P.A.P, 1950, Notes on continuous stochastic phenomena. Biometrika, 37, 17-23

Openshaw, S. and RAO, L., 1995, Algorithms for reengineering 1991 Census geography. Environment and Planning A 27, 425--446 
International Journal of Geographical Information Science Submission

Peucker, T. K. and Chrisman, N., 1975, Cartographic data structures. The American Cartographer, 2, 55-69

Rhind D., Cole K., Armstrong M., Chow L. and Openshaw S., 1991, An on-line, secure and infinitely flexible database system for the national census of population. Working Paper 14, South East Regional Research Laboratory, Birkbeck College, University of London.

Salazar-Gonzalez, J.J., Lowthian, P., Young, C., Merola, G., Bond, S. And Brown, D., 2004, Getting the best results in controlled rounding with the least effort. Privacy in statistical databases, Lecture notes in computer science, Springer-Berlin, Vol 3050

Shlomo, N., 2005, Assessment of statistical disclosure control methods for the 2001 UK Census. UNECE/Eurostat Work Session on Statistical Data Confidentiality, 9-11 Nov 2005, Geneva.

Shlomo, N., 2006, Statistical disclosure control methods for census frequency tables. Southampton Statistical Sciences Research Institute, Methodology working paper M06/03.

TOBLER, W. R. 1970. A computer movie simulating urban growth in the Detroit region. Economic Geography 46, 234-240 
International Journal of Geographical Information Science Submission

VAnWey, L.K., Rindfuss, R.R., GutManN, M.P., ENTwisle, B., and BALK, D.L, 2005, Confidentiality and spatially explicit data: concerns and challenges. Proceedings of the National Academy of Sciences of the United States of America: Spatial Demography Special Feature, 102 (43) Available online at: http://www.pnas.org/cgi/reprint/102/43/15337

Wallace, M., Charlton, J. and Denham, C., 1995, The new OPCS area classification. Population Trends, 79, 15--30

Willenborg, L. and De WAAL, T, 2001, Elements of Statistical Disclosure Control, Lecture Notes in Statistics , 155 (Springer Verlag: New York)

WiLliamson, P., BIRKIN, M., and REES, P. 1998, The estimation of population microdata by using data from small area statistics and samples of anonymised records. Environment and Planning A, 785-816

WiLliamson, P. and VoAs, D., 2000, An evaluation of the combinatorial optimization approach to the creation of synthetic microdata. International Journal of Population Geography, 349-366

ZAYATZ, L., 2003, Disclosure limitation for Census 2000 tabular data. Joint UNECE/Eurostat work session on statistical data confidentiality, 7-9 April 2003, Luxembourg, working paper 15. 
International Journal of Geographical Information Science Submission

ZAYATZ, L., 2006, Disclosure avoidance practices and research at the US Census Bureau: An Update. US Census Bureau Research Report Series. Available online at: http://www.census.gov/srd/papers/pdf/rrs2005-06.pdf

ZIMMERMAN, D.L. and PAVLIK, C., 2006, Quantifying the effects of mask metadata disclosure and multiple releases on the confidentiality of geographically masked health data. Technical Report 369, University of Iowa, Department of Statistics and Actuarial Science; Available online at: http://www.stat.uiowa.edu/techrep/tr369.pdf 